\title{
THE PLANE OF THE KUIPER BELT
}

\author{
Michael E. Brown \\ Division of Geological and Planetary Sciences, California Institute of Technology, Pasadena, CA 91125; mbrown@caltech.edu \\ AND \\ Margaret Pan \\ Department of Astronomy, California Institute of Technology, Pasadena, CA 91125; mpan@astro.caltech.edu \\ Received 2003 July 30; accepted 2003 December 23
}

\begin{abstract}
We present a robust method for measuring the effective plane of the Kuiper belt. The derived plane has an inclination with respect to the ecliptic of 1.86 and an ascending node of 81.6 , with a $1 \sigma$ error in pole position of the plane of 0.37 . The plane of the Kuiper belt is inconsistent with the invariable plane, the plane of Jupiter, and the plane of Neptune at the greater than $3 \sigma$ level. Using linear secular perturbation theory, we show that the plane of the Kuiper belt is expected to oscillate about the position of the invariable plane with a period of 1.9 million years and an amplitude of 1.2. The present predicted position of the plane of the Kuiper belt has an inclination with respect to the ecliptic of 1.74 and an ascending node of $86^{\circ} .7$, within 0.20 of our measured position.
\end{abstract}

Key words: Kuiper belt — solar system: general

\section{INTRODUCTION}

The orbital distributions of bodies in the Kuiper belt hold important clues to the formation and evolution of the outer solar system (Morbidelli \& Brown 2004). One important dynamical property of the Kuiper belt that has yet to be exploited is its overall plane. The plane of the Kuiper belt is affected by the total angular momentum of the solar system, recent stellar encounters, and unseen distant masses in the outer solar system. Early observers of the Kuiper belt implicitly assumed that the plane of the Kuiper belt was the plane of the ecliptic and that the peak concentration of objects would therefore be in the ecliptic (Jewitt \& Luu 1995; Irwin, Tremaine, \& Żytkow 1995; Gladman et al. 1998; Chiang \& Brown 1999). Several authors subsequently suggested that the invariable plane - the plane of the average angular momentum of the solar system-is more appropriately considered the plane of the Kuiper belt (Hahn 2000; Levison \& Stern 2001; Allen, Bernstein, \& Malhotra 2002). Despite the importance for searches and interpretations of knowing the correct plane, no accurate measure of the position of the plane of the Kuiper belt has been made. The only attempt to date at extracting a measurement of the plane has been from Collander-Brown et al. (2003), who examined the average angular momentum of subsets of known Kuiper belt objects but concluded that the plane's position could not be accurately determined in that manner.

We present here a new and robust method for determining the plane of the Kuiper belt that makes optimal use of the discovery information from all known Kuiper belt objects. We then examine outer solar system dynamics to determine the expected plane of the Kuiper belt given perturbations from all presently known planetary bodies. We finally conclude with a discussion of the comparison between the measured and expected plane of the Kuiper belt and the implications for the outer solar system.

\section{METHOD AND RESULTS}

\subsection{Measurement of the Plane}

For any given patch of sky on the celestial sphere, the average directional orbital velocity vector of all Kuiper belt objects within that patch must be parallel to the plane of symmetry of the Kuiper belt. It thus follows that this plane of symmetry - which we define to be the overall plane of the Kuiper belt - could hypothetically be determined uniquely by taking any two different patches in the sky, measuring the plane-of-sky orbital velocity vectors (which, in practice, simply requires measurement of the inclination) of objects in these patches, and taking the cross product of the two average directional velocity vectors to determine the pole of the plane of the Kuiper belt. There are no observationally induced biases in this hypothetical method; selection of any two patches of the sky will give precisely the same result. The difficulty with this method is, of course, that to accurately measure the average velocity vector in one spot on the sky requires that a large number of objects must be found in a small location, which is prohibitive.

A logical and practical extension to this method is easily implemented, but potential observational biases exist and must be examined. If every Kuiper belt object across the sky were known, we could determine the plane of the Kuiper belt either by trivially finding the average plane of the location of all of the objects or equivalently by finding the plane that, on average, best goes through all of the directional velocity vectors of the objects. In the practical case, we do not know all Kuiper belt objects, only some biased subset, and taking the average plane of the observed objects is fraught with unknowable and likely severe biases. As an extreme example, imagine a subset of Kuiper belt objects (KBOs) discovered exclusively at high ecliptic latitude and in one longitudinal hemisphere of the sky. The average plane of these objects will be severely biased by the latitudes and longitudes of the discovery positions. If we instead find the plane that best fits the directional velocity vectors of the objects, we have an estimate of the plane of the Kuiper belt that is almost unaffected by biases caused by the discovery positions. Even in our severe case described above, the average of all of the velocity vectors is still in the plane of the Kuiper belt even when the observational longitudes and latitudes are extremely biased. We present extensive Monte Carlo testing below to verify that this method indeed works in a manner unbiased by 
discovery location for the samples that we use in the real Kuiper belt.

We apply this method of finding the plane of the Kuiper belt to all objects known as of 2003 July 1 . We define our initial sample to include 728 objects detected at heliocentric distances greater than $30 \mathrm{AU}$. We include in our sample objects of all classes and orbital solution qualities, as our method relies only on the direction of motion, found from the inclination, which is adequately determined for almost all objects even in short series of observations (Brown 2001). Figure 1 shows for reference the ecliptic longitude and latitude at the time of discovery of the KBOs in our sample. The location of KBO discoveries clearly shows the expectations of the observers: most searches and thus discoveries appear centered on the invariable plane or on the ecliptic plane. For each object in our sample, we determine the direction of the plane-of-sky orbital velocity vector from the object position, inclination, and ascending node. These velocity vectors are shown in Figure 2. Owing to the generally extremely well determined inclinations and ascending nodes, the errors in the direction vectors are small and do not affect the following results. On average, these velocity vectors are parallel to the plane of the Kuiper belt. In the raw data it is easy to see that the plane of the Kuiper belt is displaced from the ecliptic.

To find the plane that is the best fit to all of the velocity vectors, we find the plane that minimizes the sum of the absolute values of the distances from each vector to the plane. We use a least-absolute-value fit rather than a more traditional least-squares fit because a least-squares fit makes the implicit assumption that the data are normally distributed about the true result. A least-squares fit is thus strongly weighted by (what should be extremely rare) outliers. Our data are naturally distributed much more broadly than a normal distribution, so a least-squares fit would not give an appropriate result. A least-absolute-value fit gives less weight to apparent outliers and is more appropriate for our data.

We find that the best fit to the apparent plane of the Kuiper belt has an angle with respect to the ecliptic of 1.86 and an ascending node of 81.6 . A projection of that plane onto the

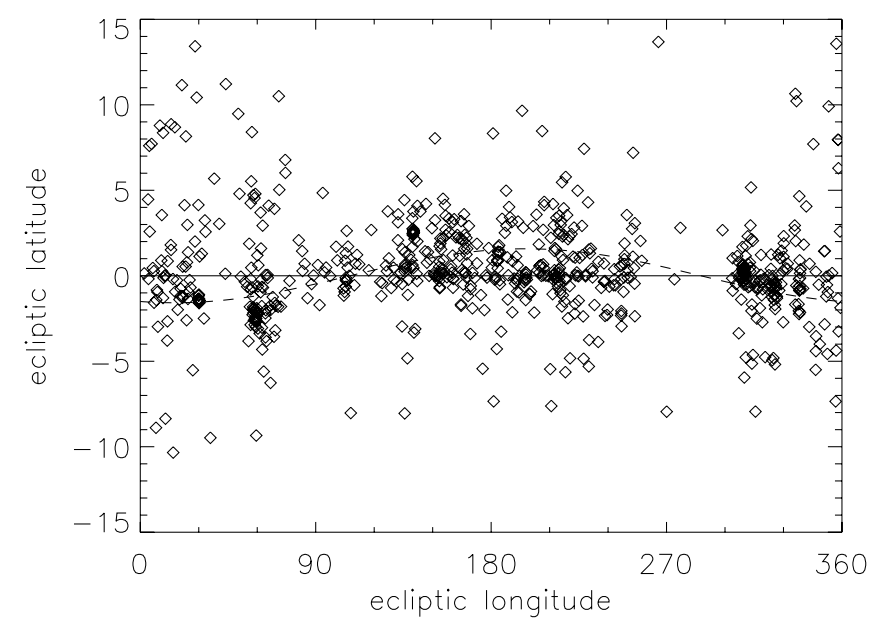

Fig. 1.-Ecliptic longitude and latitude (in degrees) at time of discovery for all KBOs discovered as of 2003 July 1 at heliocentric distances greater than $30 \mathrm{AU}$. The $16 \mathrm{KBOs}$ found at ecliptic latitudes between $15^{\circ}$ and $45^{\circ}$ are not shown. The dashed line shows the location of the invariable plane. Discoveries have been concentrated along the ecliptic plane and along the invariable plane, the two most heavily searched areas.

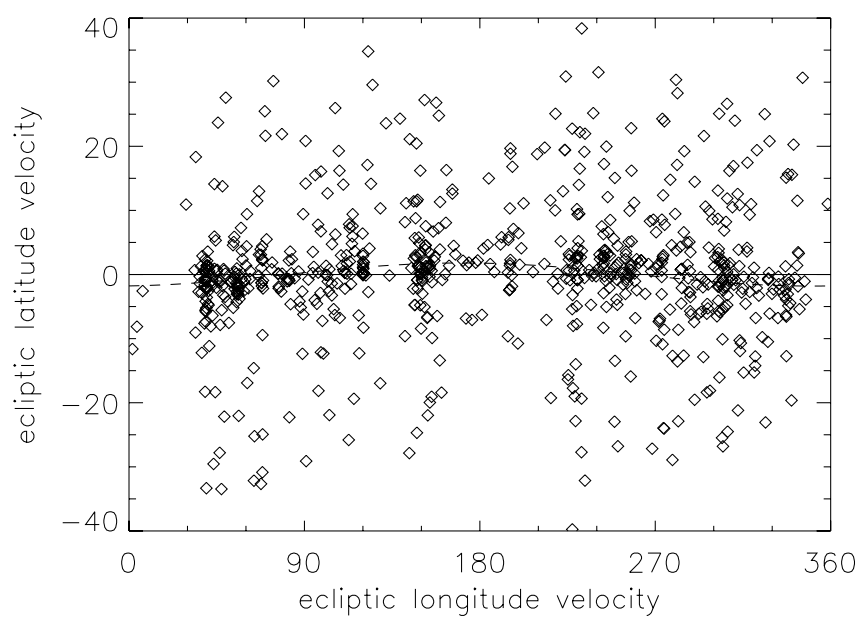

FIG. 2. - Orbital plane-of-sky velocity direction vector (in degrees) for the $\mathrm{KBOs}$ in our sample. The concentration of velocities away from the ecliptic is apparent in this plot. The dashed line shows the plane that best fits the velocity data in a least-absolute-value sense. The plane has an ascending node of 81.6 and an inclination to the ecliptic of 1.86 .

velocity vectors (Fig. 2) shows that the fit correctly accounts for the visual impression of the average velocity plane.

\subsection{Monte Carlo Validation}

To determine the validity of and the error bars on our analysis, we turn to Monte Carlo simulation of the entire process. We first assume a fixed plane to the Kuiper belt and create a model Kuiper belt with 100,000 objects selected to have random inclinations drawn from an inclination distribution determined with the method of Brown (2001) and random true anomaly between $0^{\circ}$ and $360^{\circ}$. For every KBO in our true data sample, we select a modeled KBO that is at the latitude and longitude where the true KBO was discovered. In this manner, we select a modeled Kuiper belt with a known plane that has the same number of objects discovered in the same places and the same overall inclination distribution as the real Kuiper belt, but with a different set of objects. We can then use our analysis to measure the plane of this simulated Kuiper belt and compare the results with the actual plane selected for the simulation. Repeating this technique multiple times allow us to develop statistics of the distribution of the measured plane for any true plane.

In our first simulation, we fix the plane of the Kuiper belt to be the invariable plane, and we perform 1000 separate simulations. Figure 3 shows the measured pole positions of the 1000 simulated Kuiper belts. The average of the 1000 measurements is within 0.01 of the invariable plane, demonstrating that the method is, on average, adept at accurately measuring the true plane of the Kuiper belt. The spread in the measured poles shows the accuracy of any single measurement, including our real measurement. The 1000 individual pole measurements are distributed roughly spherically symmetrically around the true pole, and the circles containing $84.1 \%, 97.7 \%$, and $99.9 \%(1,2$, and $3 \sigma)$ of the points have radii of $0.37,0.52$, and 0.75 , respectively. Only one of the 1000 Monte Carlo simulations has a measured pole position as distant from the invariable plane as is our measured Kuiper belt pole position. We can thus rule out the invariable plane as the true plane of the Kuiper belt at the greater than $3 \sigma$ level. 


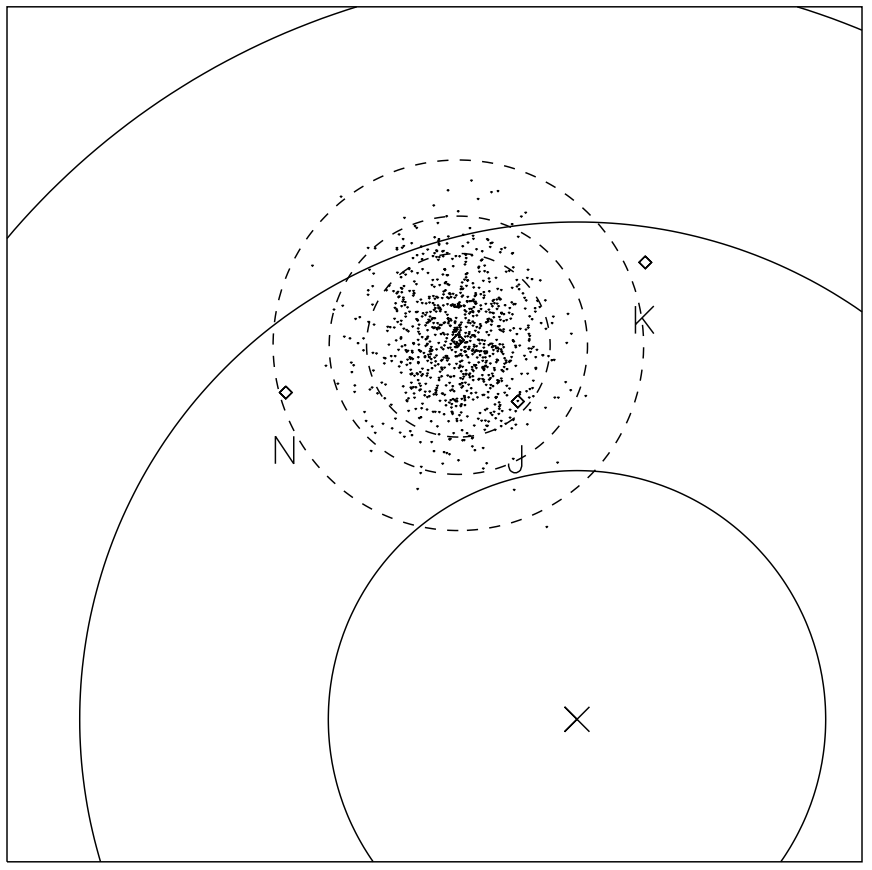

FIG. 3.-Pole positions of the planes of Neptune $(\mathrm{N})$, Jupiter (J), the measured Kuiper belt $(\mathrm{K})$, and the invariable plane (unlabeled diamond) compared with the pole position measured for the plane in 1000 simulated Kuiper belts where the plane was fixed as the invariable plane. The cross shows the ecliptic north pole, and the large circles are separated by $1^{\circ}$ of latitude. The dashed circles show the radii at which $84.1 \%, 97.7 \%$, and $99.9 \%$ of the points are within the circle, showing the 1,2 , and $3 \sigma$ error in the measurement of $0.36,0^{\circ} .53$, and 0.72 , respectively.

Similar Monte Carlo simulations with the plane positions in different locations within a few degrees all show a nearly identical spread in measurements. In Figure 4, we show the distribution of pole positions that would be measured if the true plane were at the position of the plane that we measured above for the real Kuiper belt. The 1,2, and $3 \sigma$ error circles have radii of $0.36,0.53$, and $0^{\circ} .72$. We will adopt the above numbers as the error bars on our measurement, though we point out that the rigorous method to determine the error bars on our measurements is to assume a large number of different pole positions and calculate the probability that a real pole a certain distance away would yield the above measured value. We expect, however, that these two different methods would give essentially identical results.

\subsection{Planes of Subsets of Objects}

The same method can be used to separately determine the plane of any subset of objects in the Kuiper belt. If we take the 70 multiopposition Plutinos as our sample, for example, we find a plane with an ascending node of $120^{\circ}$ and an inclination of 1.2 - much closer to the plane of Neptune than the previous measurement. Monte Carlo simulation, however, shows that this measurement is almost meaningless. The small number of objects, combined with the very wide spread in inclination of the Plutinos (Brown 2001), makes the 1, 2, and $3 \sigma$ error bars on the measurement $7^{\circ}, 10^{\circ}$, and $15^{\circ}$, respectively. Measurement of the plane of the scattered Kuiper belt objects or of any other small subset of objects gives equally unreliable results. We therefore will only consider the average plane of the entire Kuiper belt, with the caveat that different dynamical classes and objects at differing semimajor axes could indeed have different planes.

\section{PREDICTED PLANE}

The plane of the Kuiper belt is frequently assumed to be the invariable plane of the solar system. The measurement of the position of the plane of the Kuiper belt above differs significantly from the invariable plane. Several dynamical effects could change the plane of the Kuiper belt, including unseen masses in the outer solar system and recent stellar encounters. Below, we attempt to better understand the expected plane of the Kuiper belt by examining the magnitude of the gravitational perturbations to KBOs from the major planets, and then we calculate the combined effect of all planets on the Kuiper belt. We find that the assumption that the plane of the Kuiper belt should be the invariable plane is overly simplistic and that the current expected plane of the Kuiper belt differs significantly from the invariable plane.

\subsection{Neptune versus Jupiter}

Objects in the Kuiper belt are likely to be most affected gravitationally by Jupiter, the most massive planet in the solar system, or by Neptune, the planet closest to the Kuiper belt. The magnitudes of the effects of these planets can be considered by calculating the average gravitational effect of each planet. One method by which to calculate this effect is to consider all planets as if their masses were smeared into annuli following their orbits around the Sun. We numerically integrate the perpendicular component of the noncentral force affecting a 44 AU Kuiper belt object and find that equal-mass objects at the location of Jupiter, Saturn, Uranus, and Neptune exert relative precessional forces in the ratio 1.0, 3.8, 20.9, and 90.6, respectively. Multiplying these magnitudes by the masses of these planets shows that the relative effects on a 44 AU Kuiper belt object for the major planets are 1.0, 1.1,

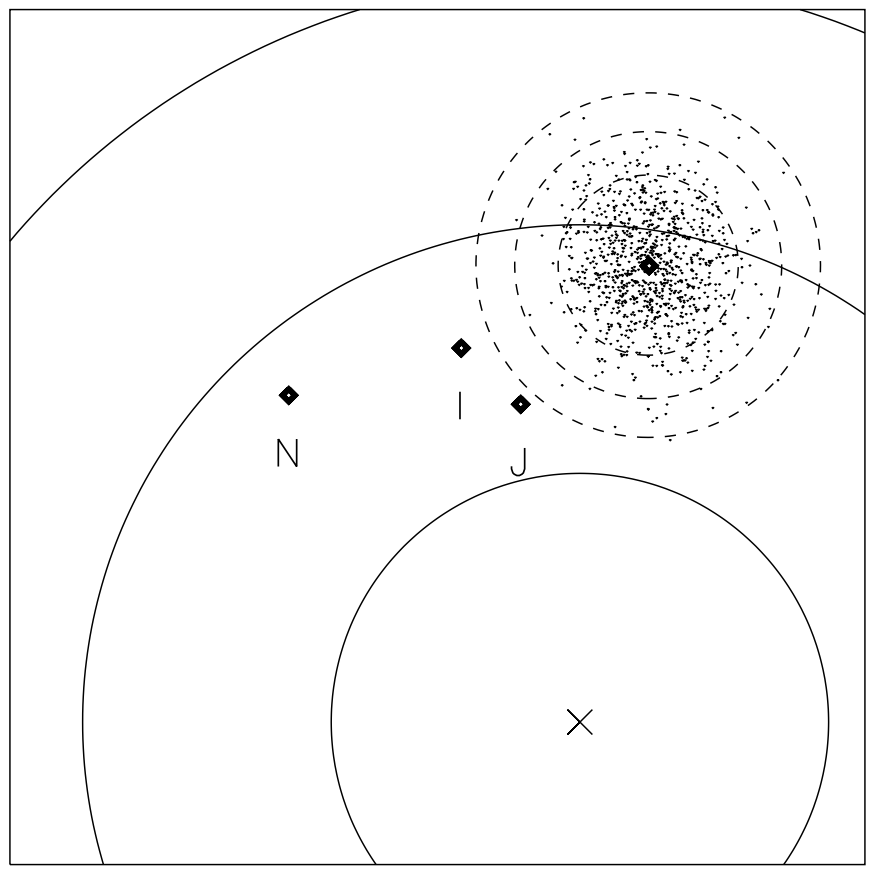

FIG. 4.-Comparison of the pole positions of the planes of Neptune, Jupiter, the invariable plane, the measured plane of the Kuiper belt, and 1000 simulated measurements where the plane was fixed at the measured plane. The dashed lines again show the 1,2 , and $3 \sigma$ error bars in the measurement. The measured plane of the Kuiper belt is more than $3 \sigma$ distant from the invariable plane and the planes of Neptune and Jupiter. 


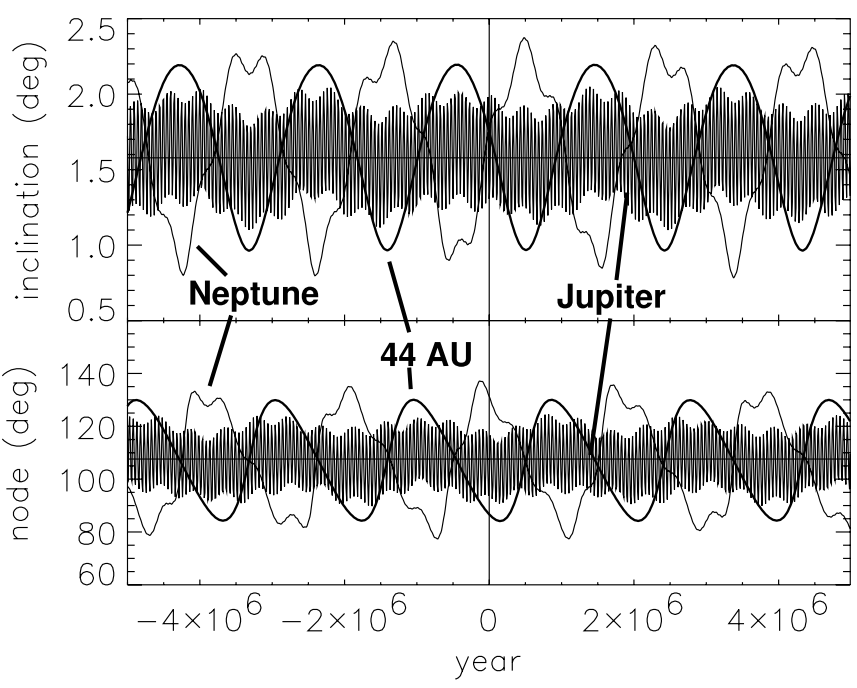

Fig. 5.- Inclination and ascending node of the orbits of Jupiter, Neptune, and the forced plane of a test particle with a semimajor axis of $44 \mathrm{AU}$, as a function of time, referenced to the ecliptic, from $5 \mathrm{Myr}$ ago until $5 \mathrm{Myr}$ from now. The solid line at 1.58 inclination and $107^{\circ} .6$ ascending node shows the location of the invariable plane. The planes of Jupiter and Neptune and the Kuiper belt all oscillate about the invariable plane.

0.9 , and 4.9 , respectively. We thus see that Neptune should exert the largest control over the Kuiper belt.

\subsection{Orbital Perturbations}

While the simple calculation above provides insight into the competing forces affecting particles in the Kuiper belt, a more detailed calculation is required to predict the location of the plane of the Kuiper belt. Small bodies in the solar system undergo orbital perturbations owing to the combined gravitational effects of all major planets. Except for regions near resonances, these perturbations can be divided into two components. First, all bodies undergo precession of their perihelia and longitudes of ascending nodes. The instantaneous plane about which the nodal precession occurs is called the forced plane (or the Laplace plane, in studies of planetary satellites), and it is this plane that is instantaneously the plane of the Kuiper belt. Second, the forced plane will circulate about the invariable plane with a different timescale. This second perturbation is caused by the mutual interactions of all of the major planets and the perturbations of their own orbital planes.

To find the expected plane of the Kuiper belt, we first use the first-order secular perturbation theory of Brouwer \& van Woerkom (1950), which determines the mutual perturbations of all planets on each other. With these perturbations known, we then use the extension of Murray \& Dermott (1999) to calculate the location of the forced plane for any object in the solar system as a function of time. Figure 5 shows the location of the forced plane of a test particle with a semimajor axis of $44 \mathrm{AU}$ (the median semimajor axis of the objects in our sample) compared with the planes of Jupiter and Neptune and with the invariable plane. All of the planes circulate about the invariable plane. The plane of the 44 AU test particle circulates about the invariable plane with an amplitude of 1.2 and a period of 1.9 million years. Figure 6 shows a polar projection of the forced pole position of plane of the planes of Jupiter, Neptune, and the 44 AU particle for a 10 million year period. The circulation of the planets and the test particle about the invariable plane is apparent.

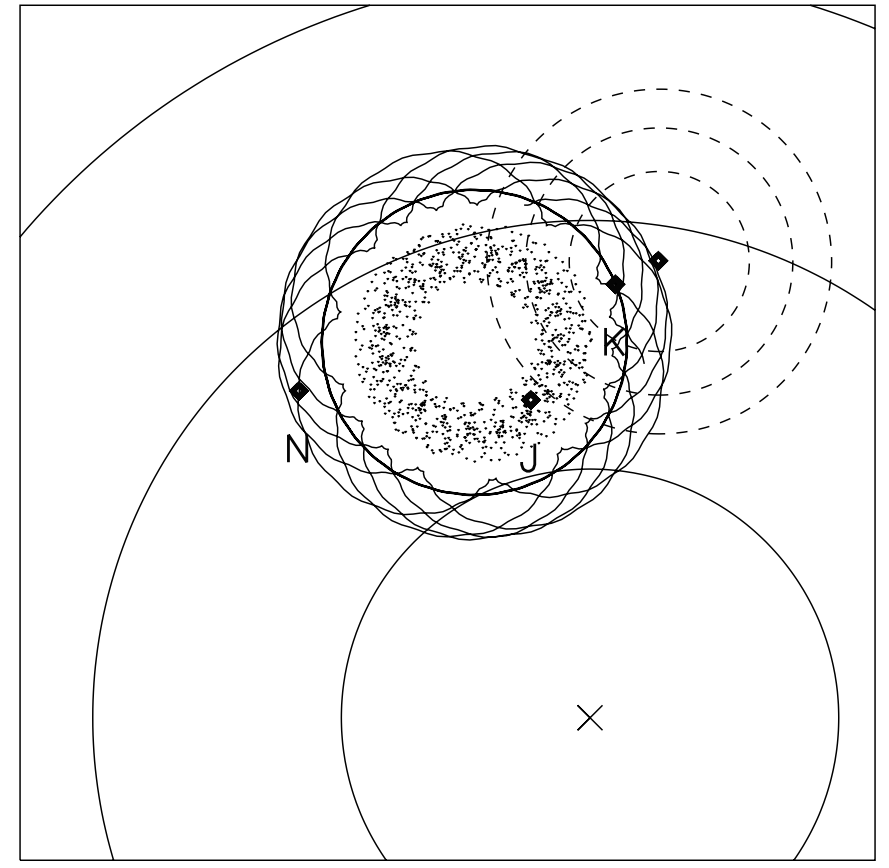

Fig. 6.-Poles of the planes of the orbits of Jupiter (dots), Neptune (thin line), and the Kuiper belt (thick line) over a $10 \mathrm{Myr}$ period. The current planes of Neptune and Jupiter, the forced plane of the $44 \mathrm{AU}$ test particles, and the measured plane of the Kuiper belt (with 1,2, and $3 \sigma$ error circles) are shown as diamonds. The measured plane is more than $3 \sigma$ distant from the planes of Jupiter and Neptune but is within 0.20 of the plane expected from secular perturbation theory.

Figure 7 shows the location of the present-day forced plane for any test particle placed in the solar system. As required, in the region near each planet the forced plane is equal to the plane of that planet. At the marked locations of secular resonances, secular perturbation theory fails (and few solar system objects exist in stable orbits). In the region of the classical Kuiper belt, the forced plane slowly moves closer to the invariable plane with increasing semimajor axis.

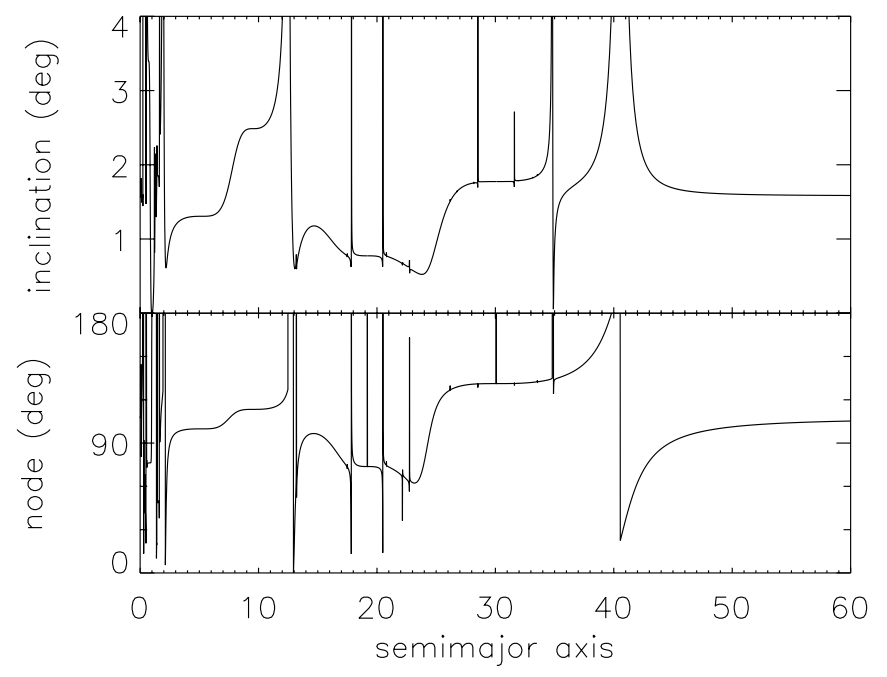

FIG. 7.- Inclination and ascending node with respect to the ecliptic plane of the forced plane of a test particle as a function of semimajor axis. The forced plane is generally similar to that of the planet closest to the test particle except near secular resonances, where the inclinations can get highly excited and linear secular perturbation theory fails. 
The expected plane of the Kuiper belt is difficult to determine precisely through this first-order secular perturbation theory. Objects in mean motion resonances and objects with high eccentricity or inclination - which includes a substantial fraction of the population - are potentially poorly described by linear secular perturbation theory. In addition, Figure 7 shows that the theory predicts significantly different planes for different regions of the Kuiper belt. Nonetheless, we will take as the predicted position of the Kuiper belt the plane of the test particle at $44 \mathrm{AU}$, which is the median semimajor axis of all of the objects in our sample. This plane has an inclination with respect to the ecliptic of 1.74 and an ascending node of $86^{\circ} .7$, 0.20 different from our measured Kuiper belt plane. We thus conclude that secular perturbation theory adequately predicts the true location of the apparent plane of the Kuiper belt and no compelling reason exists to consider other, more exotic effects.

\subsection{Neptune and the Kuiper Belt}

As seen in Figure 5, the plane of an object at 44 AU circulates about the invariable plane with the same period as Neptune but with the opposite phase. This initially counterintuitive result can be understood by examining the forcing of Neptune and the effect of the secular resonance at 40.4 AU. As shown above, the precession of the forced nodes of objects in the Kuiper belt is affected primarily by Neptune. The period of the forcing by Neptune is the period of Neptune's nodal precession, which is $1.9 \mathrm{Myr}$. The response to this forcing can be seen in Figure 8, which shows the angle with respect to Neptune of the node of the forced plane and the forced inclination with respect to the invariable plane as a function of semimajor axis.

The amplitude and phase of the response to this forcing can be understood in the context of a forced simple harmonic oscillator (SHO). When an SHO is forced at a frequency much lower than the resonant frequency, the response is an in-phase

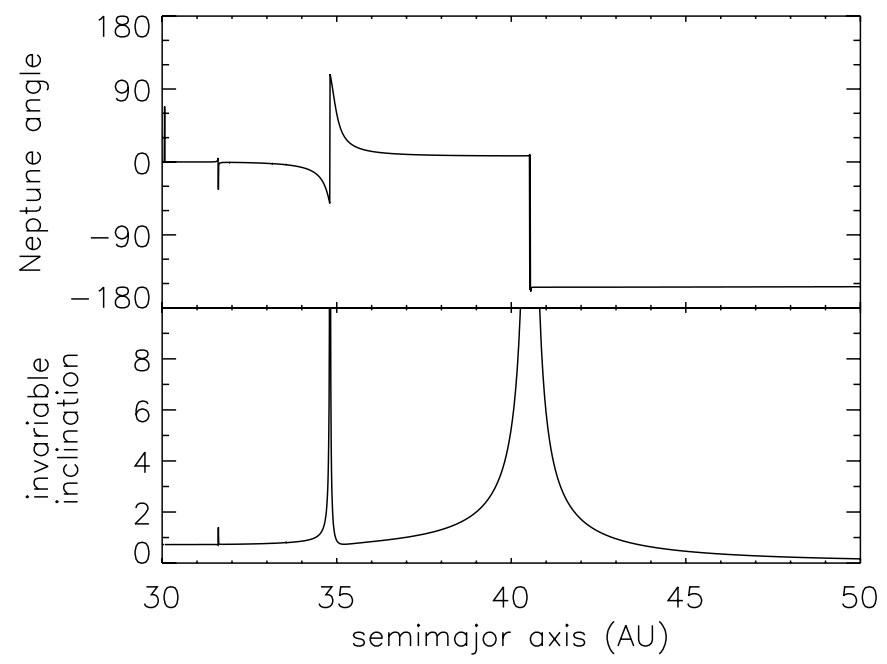

FIG. 8.-Plane of a test particle as a function of semimajor axis compared with the plane of Neptune. The ascending node and inclination are measured relative to the invariable plane. Interior to the $\nu_{18}$ secular resonance at 40.4 AU, the test particle oscillates about the invariable plane in phase with Neptune with an inclination similar to Neptune's. Near the secular resonance, the inclination of the test particle becomes highly excited. Outside the resonance the test particle circulates $180^{\circ}$ out of phase from Neptune and with a decreasing inclination. This behavior is that expected from a simple harmonic oscillator being forced at the precession frequency of Neptune. oscillation with an amplitude nearly equal to the forcing amplitude. Near the resonant frequency the amplitude of oscillation increases dramatically. At a frequency much higher than the resonant frequency, the response is an out-of-phase oscillation with a amplitude decreasing as the forcing frequency increases.

For the case of the Kuiper belt and the forcing by Neptune, the resonant frequency occurs when the $1.9 \mathrm{Myr}$ period of Neptune's precession is equal to the natural precession time of an object about its forced plane. This equality, which defines the location of the $\nu_{18}$ secular resonance, occurs at $40.4 \mathrm{AU}$. At smaller semimajor axes, Neptune's precession frequency is smaller than the natural precession frequency, so the response is an in-phase oscillation of magnitude equal to the magnitude of Neptune's forcing. At larger semimajor axes, such as the location of most of the objects in the Kuiper belt, Neptune's precession frequency is larger than the natural precession frequency, so the response is an out-of-phase oscillation with an amplitude that decreases with increasing semimajor axis. The current location of the plane of the Kuiper belt is thus seen as a natural consequence of high-frequency forcing by the nodal precession of Neptune.

\section{CONCLUSION}

The apparent plane of the Kuiper belt is found to have an inclination of 1.86 and an ascending node of 81.6 with a $1 \sigma$ error in the pole position of 0.37 . This plane is offset by 1.86 from the ecliptic and by 0.82 from the invariable plane. This significant tilt of the apparent plane away from the two planes usually assumed as the Kuiper belt plane is an expected consequence of the forcing by the 1.9 million year precession of the node of Neptune at 0.7 from the invariable plane. The Kuiper belt has no one true plane, as secular perturbations warp the plane of objects as a function of semimajor axis.

This newly determined Kuiper belt plane should be used for all calculations of inclinations and ascending nodes and can make an important difference in the calculation of quantities such as the inclination distribution of the Kuiper belt. For example, using the method of Brown (2001) to determine the inclination distribution of the classical Kuiper belt and assuming that the plane of the Kuiper belt is the plane measured gives a best-fit inclination distribution of $\sin i$ times two Gaussians with $\sigma=1.3$ and $\sigma=12.0$. In contrast, when the ecliptic is used as the assumed plane of the Kuiper belt, the narrowly peaked component is almost $50 \%$ wider. Similar important effects can be seen in reexamining results such as the correlation between inclinations and colors (Trujillo \& Brown 2002) and inclinations and absolute magnitudes (Levison \& Stern 2001; Trujillo \& Brown 2004) seen in the classical Kuiper belt.

The method developed here for determining the plane of the Kuiper belt is robust and applicable to any dynamical or physical subset of objects. In the future, as thousands of Kuiper belt objects begin to be discovered, even tighter error bars on the location of the overall plane, and good determinations of the planes of different subsets of objects, will allow the continued exploration of the dynamical structures in the outer solar system.

We thank Chad Trujillo and Re'em Sari for inspirational conversations. This research is supported by a PECASE grant from NASA Planetary Astronomy. 


\section{REFERENCES}

Allen, R. L., Bernstein, G. M., \& Malhotra, R. 2002, AJ, 124, 2949

Brouwer, D., \& van Woerkom, A. J. J. 1950, The Secular Variations of the Orbital Elements of the Principal Planets (Washington: GPO)

Brown, M. E. 2001, AJ, 121, 2804

Chiang, E. I., \& Brown, M. E. 1999, AJ, 118, 1411

Collander-Brown, S. J., Melita, M. D., Williams, I. P., \& Fitzsimmons, A. 2003, Icarus, 162,22

Gladman, B., Kavelaars, J. J., Nicholson, P. D., Loredo, T. J., \& Burns, J. A. 1998, AJ, 116, 2042

Hahn, J. M. 2000, in Lunar and Planetary Science XXXI (Houston: Lunar Planet. Inst.), No. 1797
Irwin, M., Tremaine, S., \& Żytkow, A. N. 1995, AJ, 110, 3082

Jewitt, D. C., \& Luu, J. X. 1995, AJ, 109, 1867

Levison, H. F., \& Stern, S. A. 2001, AJ, 121, 1730

Morbidelli, A., \& Brown, M. E. 2004, in Comets II, ed. M. Festou, H. U. Keller, \& H. A. Weaver (Tucson: Univ. Arizona Press), in press

Murray, C. D., \& Dermott, S. F. 1999, Solar System Dynamics (Cambridge: Cambridge Univ. Press)

Trujillo, C. A., \& Brown, M. E. 2002, ApJ, 566, L125

2004, Earth Moon Planets, in press 